Dochead : Maladies infectieuses

\title{
Épidémiologie des infections en transplantation rénale
}

\section{Infectious complications after kidney transplantation}

Anne Scemla ${ }^{a *, b}$, Victoria Manda a,b

${ }^{a}$ Service de néphrologie et transplantation adulte, hôpital Necker Enfants Malades, Assistance publique-hôpitaux de Paris, 149 rue de Sèvres, 75015 Paris, France

${ }^{\text {b }}$ Université Paris Descartes Sorbonne Paris Cité, 12 rue de l'École-de-Médecine, 75006 Paris, France

*Auteur correspondant ; téléphone : +33 144381992 ; e-mail : anne.scemla@aphp.fr

\section{Liens d'intérêt}

Anne Scemla ne déclare pas de lien d'intérêt.

Victoria Manda ne déclare pas de lien d'intérêt. 


\section{Introduction}

En transplantation rénale, les infections sont la première ou deuxième cause de mortalité, et sont responsables d'une morbidité élevée, incluant hospitalisations et dégradation de fonction rénale ou perte de greffon.

L'évolution des traitements immunosuppresseurs, des prophylaxies anti-infectieuses, et surtout des caractéristiques des patients transplantés ont conduit à une modification de l'épidémiologie des infections ces dernières décennies. Les patients sont en effet plus âgés, ont plus de comorbidités, sont plus immunisés, et reçoivent pour une partie d'entre eux une nouvelle transplantation, ce qui est responsable d'une imunosuppression accrue. Enfin, les patients transplantés voyagent plus ou sont plus souvent issus de pays ayant des maladies endémiques, ce qui conduit à l'émergence de nouvelles pathologies.

\section{2. Épidémiologie générale}

Les complications infectieuses sont fréquentes et graves chez les patients ayant eu une greffe de rein. Selon les études, il est estimé qu'elles surviennent chez 45 à $70 \%$ des patients $(1,2)$. Elles représentent la première ou deuxième cause de mortalité et d'hospitalisation en transplantation rénale (3). Les micro-organismes responsables des infections sont les bactéries, communautaires ou nosocomiales (23 à $46 \%$ dans les cohortes américaines), les virus (17 à 18\%), et les champignons et parasites (5\%) $(1,4)$.

Les infections sont plus fréquentes la première année suivant la transplantation, et notamment le premier mois, en raison d'infections liées aux soins et à l'intensité de l'immunosuppression. Puis leur incidence diminue progressivement au fil des années.

Les infections urinaires sont de loin les plus fréquentes, surtout la première année, suivies des infections respiratoires, de la peau et des tissus mous, et gastro-intestinales $(3,4)$. Le premier mois suivant la transplantation, les infections urinaires hautes, les colites à clostridium, les infections du site opératoire, les infections de cathéter prédominent. L'année qui suit la transplantation, des infections urinaires hautes et des pneumopathies surviennent principalement, mais également les maladies à cytomegalovirus (CMV) tardives, les néphrites à BK virus et les tuberculoses. Au delà, les infections communautaires restent prédominantes, mais d'autres infections opportunistes peuvent survenir, notamment les zonas, les infections fongiques invasives, et les mycobactéries atypiques.

Les facteurs de risque d'infection bien démontrés sont l'âge, le sexe féminin (risque accru d'infections urinaires), le temps passé en dialyse, le diabète et la survenue de rejet. L'incidences des infections n'a pas diminué entre les années 1990 et 2000, et ce malgré les prophylaxies contre la pneumocystose et le CMV (4). 


\section{Infections dérivées du donneur}

Certaines infections du donneur peuvent être transmises au receveur lors de la transplantation. Ces infections, comme les infections à $\mathrm{CMV}$, la tuberculose, certaines infections fongiques endémiques (histoplasmose, coccidioidomycose) sont le plus souvent latentes dans les tissus. Des pathogènes rares, souvent mortels, comme le West Nile virus ou la rage, ont aussi été au receveur par le donneur. Si la présence, chez le donneur, d'une infection par le virus de l'immunodéficience humain (VIH) contreindique la greffe, il n'en est pas de même des organes des donneurs atteints d'hépatites B et C, qui sont réservés à certains receveurs. Toutes ces infections virales, si elles sont méconnues, peuvent être transmises au receveur.

\section{Infections communautaires}

\subsection{Infections urinaires hautes}

La pyélonéphrite aiguë est la première complication infectieuse après transplantation rénale, et ce à tout moment après la transplantation. Elle concernerait 13 à 20\% des patients la première année (5-7). Escherichia coli est le principal pathogène identifié (5).

La bactériurie asymptomatique, considérée jusque récemment comme fréquente, serait en fait très rare, avec une prévalence de 3 à $5 \%$ chez les patients greffés depuis plus de 2 mois. Ces bactériuries asymptomatiques ne semblent pas nécessiter de traitement (8).

Les facteurs de risque principaux en sont le sexe féminin, l'âge, le diabète, l'insuffisance rénale, le recours à la dialyse et la présence de sonde $(5,6)$. L'infection à CMV pourrait également en être un facteur de risque (5).

Selon plusieurs études, la survenue de pyélonéphrite serait associée à une dégradation de la fonction rénale, voire à une de perte de greffon $(5,7)$

\subsection{Pneumopathies}

Les pneumopathies représentent la deuxième cause d'infection en transplantation rénale et concerneraient entre 5 et $10 \%$ des patients (9). Comme dans la population générale, dans 70 à $80 \%$ des cas, l'étiologie de la pneumopathie est indéterminée. Lorsqu'une cause est retrouvée, il s'agit principalement de bactéries (pneumocoque, haemophilus, entérobactéries, mycobactéries), suivies des virus dont la grippe, puis des infections fongiques. L'incidence des infections respiratoires virales, dont la grippe, est probablement sous-estimée par des investigations microbiologiques insuffisantes. Ainsi, une partie de ces pneumopathies pourraient être prévenue par les vaccins ou par la prophylaxie prolongée contre la pneumocystose. 


\subsection{Bactériémies}

Elles concernent 8 à $15 \%$ des patients transplantés, et ont pour porte d'entrée principale une pyélonéphrite ou un cathéter.

\subsection{Infections hépatogastro-intestinales}

Les incidences cumulées des épisodes de diarrhée sont, d'après des données américaines de 11,5\%, $17,5 \%$ et $22,6 \%$ à 1,2 et 3 ans suivant transplantation rénale (10). La survenue de diarrhée en posttransplantation est associée à une diminution de la qualité de vie, un déclin de la fonction du greffon et un risque plus élevé de mortalité (10).

Ces 10 dernières années, de multiples études ont montré que le norovirus est la première cause infectieuse de diarrhée chez l'adulte immunocompétent, mais également en transplantation rénale. Il y serait responsable de 15 à $25 \%$ des causes de diarrhée $(11,12)$. Chez les patients transplantés, l'infection peut évoluer vers un portage chronique et les symptômes peuvent se prolonger plusieurs mois.

Les autres causes infectieuses de diarrhée sont bactériennes (Clostridium difficile, Campylobacter jejuni, Salmonella, Echerichia coli, Listeria monocytogenes), virales (CMV et autres virus entériques) et mycoparasitaires (microsporidies, cryptosporidies) (13).

Le virus de l'hépatite E est transmis par des produits d'origine animale provenant d'un animal infecté (porc, sanglier, cerf, dont abats), ou par de l'eau ou des aliments contaminés par des fèces. Certains aliments doivent donc être évités après transplantation. L'hépatite fulminante est très rare, en revanche les formes chroniques d'hépatite $\mathrm{E}$ sont fréquentes après transplantation rénale, avec un risque d'évolution rapide vers la cirrhose. L'hépatite $\mathrm{E}$ doit donc être recherchée devant toute cytolyse persistante, même modérée. Le traitement repose sur l'allègement de l'immunosuppression et la ribavirine (14)

\section{Infections opportunistes}

\subsection{Infection à cytomégalovirus (CMV)}

Le CMV est responsable d'effet directs (maladie à CMV) et indirects moins bien prouvés (rejet, infections par d'autres micro-organismes, dysfonction du greffon).

La maladie à CMV (définie par la présence de symptômes généraux et/ou de localisations tissulaires associés à une infection par le CMV) survient plus généralement la première année suivant la greffe, à l'arrêt de la prophylaxie. Elle a une incidence globale d'environ $6 \%$ chez les patients recevant une transplantation d'organe solide. Cette incidence varie en fonction du statut sérologique 
du donneur (D) et du receveur (R), l'incidence de la maladie à CMV pouvant augmenter jusqu'à 17\% chez les $\mathrm{D}+/ \mathrm{R}-(15)$.

Hormis le statut $\mathrm{D}+\mathrm{R}-$, les principaux facteurs de risque de maladie à CMV sont liés à l'immunosuppression: induction par des anticorps polyclonaux antilymphocytaires, protocoles de désensibilisation, traitement d'un épisode de rejet $(15,16)$.

\subsection{Infection à $B K$ virus}

Le BK virus est un polyomavirus latent dans l'urothélium et l'épithélium rénal. Il est responsable de néphropathies interstitielles en transplantation rénale. Son incidence a augmenté ces 20 dernières années, du fait de traitements immunosuppresseurs plus lourds, et surtout de meilleures techniques diagnostiques (17). Les néphropathies à BK virus surviennent chez 1 à 5\% des patients (18). Ainsi dans une cohorte américaine de 42838 patients ayant reçu une greffe de rein entre 2004 et 2006, l'incidence à 6 mois et 12 mois de néphropathie à BK virus était respectivement de 1,7\% et de 2,7\% (19).

Le diagnostic précoce est fait par le suivi régulier de la virémie BK l'année qui suit la transplantation, et le diagnostic de certitude se fait par la biopsie du greffon rénal.

La réduction de l'immunosuppression est le seul traitement recommandé et utile (19), ce qui peut exposer dans les suites à des épisodes de rejets. Le diagnostic tardif expose à un déclin irréversible de la fonction rénale, à une mauvaise réponse au traitement et une perte de greffon (18).

\subsection{Zona}

L'incidence du zona est augmentée en transplantation rénale. Ainsi dans une étude rétrospective, réalisée chez 612 patients transplantés rénaux, 11,2\% des patients ont développé un zona dans les 4 ans après la greffe. Le facteur de risque principal en était l'âge, chaque décennie conférant une augmentation de 1,4. Les douleurs postzostériennes concernent $24 \%$ des patients, ce qui est supérieur à la population générale, et semblent également plus intenses (20).

\subsection{Infections à Mycobacterium tuberculosis}

Le risque de tuberculose maladie est augmenté de 20 à 75 fois par rapport à la population générale après transplantation. Dans les zones de faible prévalence, comme en France, le taux de tuberculose chez les patients ayant eu une greffe d'organe varie de 0,26 à $3 \%$ selon les études et l'organe transplanté (21).

Dans une étude prospective multicentrique espagnole, sept cas de tuberculose ont été rapportés chez 2052 transplantés rénaux, soit une incidence de 358/10 000 patients par an. La mortalité attribuable à la tuberculose sur l'ensemble de la cohorte s'élevait à 9,5\%, avec une mortalité brute de 
19\% (22). Dans une cohorte française rétrospective et multicentrique de Canet, le temps écoulé entre la transplantation rénale et l'apparition d'une tuberculose maladie était de 10 mois. Les formes extrapulmonaires et disséminés représentaient $67,4 \%$ des cas (23). Ces données pourraient plaider pour le traitement des tuberculoses latentes, avant et après la greffe, comme cela est recommandé par les sociétés savantes.

\subsection{Infections à mycobactéries atypiques}

Il existe très peu de données en transplantation rénale, du fait d'une incidence faible et donc d'une difficulté à réaliser des études prospectives bien menées (24). De nombreux cas cliniques ont été rapportés. Leur incidence semble en augmentation chez les personnes âgées transplantées.

\subsection{Infections fongiques invasives}

Les infections fongiques invasives concerneraient 3 à 5\% des patients. Leur épidémiologie changeante au cours du temps et selon les pays rend leur étude difficile. Aux États-Unis, les infections fongiques invasives surviennent chez 3,8\% des patients qui ont reçu une greffe de rein (25). Les principaux pathogènes américains sont Candida, Aspergillus, Cryptococcus et les autres filamenteux. En France, l'épidémiologie est probablement différente, les pneumocystoses semblent beaucoup plus fréquentes et les champignons endémiques (histoplasmose et coccidioidomycose) très rares. Lorsqu'il existe une localisation, il faut toujours rechercher une infection disséminée.

La majorité des infections fongiques invasives apparaissent plus de 3 mois après la greffe, les infections à Candida et les aspergilloses étant les plus précoces, et les cryptococcoses plus tardives.

Les localisations des aspergilloses sont essentiellement pulmonaires, souvent bilatérales. Sous voriconazole, traitement de référence, la survie s'est considérablement améliorée, elle est à 12 mois de $60 \%$ à $80 \%$ selon les études (26). Les facteurs de risque identifiés sont la corticothérapie à forte dose et prolongée, le recours à l'hémodialyse, et la survenue préalable d'une infection opportuniste ou cancer.

L'incidence de la pneumocystose pulmonaire était de 2 à $15 \%$ avant l'ère de la prophylaxie, et a nettement diminué depuis (27). La mortalité peut atteindre 33\% (28). Le délai de survenue est tardif, car ces infections surviennent à l'arrêt de la prophylaxie, recommandée 3 à 12 mois après la transplantation. Certains centres proposent actuellement une prophylaxie à vie.

La cryptococcose représente 8 à 15\% des infections fongiques invasives en transplantation rénale. Cette infection survient en médiane dans la deuxième ou troisième année de greffe, et se présente dans deux tiers des cas sous une forme disséminée avec atteinte neuroméningée. La mortalité à 3 mois est de $14 \%$, et à 12 mois de $27 \%$. L'immunosuppression par les inhibiteurs de la calcineurine est un facteur protecteur, il ne faut donc pas l'arrêter lorsque cette infection survient (29). 
Les infections fongiques émergentes comprennent les mycoses endémiques (histoplasmose, coccidioidomycose), et les filamenteux émergents (dont les mucormycoses, fusarioses et les phaeohyphomycoses). Elles surviennent elles-aussi plus tardivement, leur médiane d'apparition étant de 343 jours (25). Les formes cutanées des filamenteux émergents sont de plus en plus décrites, et nécessitent de façon quasi constante une exérèse chirurgicale en plus du traitement antifongique.

\section{Prévention des infections}

Ces deux dernières décennies, le développement des prophylaxie anti-infectieuses a considérablement réduit la morbimortalité liée aux infections dans l'année suivant la transplantation rénale. Il s'agit de traitements préventifs, efficaces mais qui pourraient encore être mieux personnalisés, et de vaccins avant et après la transplantation, dont l'utilisation se répand progressivement.

\subsection{Prévention du CMV}

La prévention du CMV repose sur les traitements prophylactique (traitement systématique par valganciclovir ou valaciclovir) ou préemptif (traitement en cas de virémie positive). Ils peuvent être proposés avec une efficacité équivalente, pendant 3 à 4 mois après la transplantation quand le receveur est séropositif pour le CMV et pendant 6 mois lorsque le receveur est négatif est le donneur positif. Le traitement prophylactique est préféré quand l'immunosuppression est maximale (sérum antilymphocytaire, désensibilisation, greffe ABO incompatible). Lorsque le donneur et le receveur sont séronégatifs pour le CMV, une prophylaxie antiherpétique par aciclovir ou valaciclovir est recommandée pour une durée de 3 à 4 mois (30).

\subsection{Prévention de la pneumocystose}

La prévention de la pneumocystose repose principalement sur le cotrimoxazole, recommandé 3 à 12 mois après la transplantation. Certains centres proposent actuellement une prophylaxie à vie. En cas d'allergie ou leucopénie au cotrimoxazole, les aérosols mensuels de pentamidine ou l'atovaquone sont conseillés.

\subsection{Vaccination}

L'accès à la vaccination des patients qui ont reçu une transplantation est un défi majeur. Pendant des décennies, les vaccins ont été contre-indiqués chez ces patients par crainte de rejet. Il est aujourd'hui démontré que les vaccins n'augmentent pas l'incidence de rejet.

La réponse vaccinale est diminuée après la transplantation rénale, comparée aux patients ayant une maladie rénale chronique sans transplantation (31). De ce fait, il est préférable de vacciner tant que possible avant la transplantation, pour optimiser la protection vaccinale. En outre, les vaccins vivants atténués (varicella zoster virus, rougeole-oreillons-rubéole, BCG, fièvre jaune) sont strictement contre-indiqués dans le mois qui précède la greffe, après la transplantation rénale ou en cas 
d'immunosuppression en général. Il faut donc les faire, lorsque cela est nécessaire, avant la transplantation rénale (32).

De nombreuses maladies, plus fréquentes chez les patients immunodéprimés, pourraient ainsi être prévenues par la vaccination, notamment la grippe, les infections invasives à pneumocoque et le zona (32)

La grippe est responsable d'une forte morbimortalité en transplantation rénale. L'efficacité de la vaccination antigrippale saisonnière varie selon les études de 0 à $60 \%$ (33). Néanmoins, ce vaccin réduit la mortalité et les hospitalisations, il est donc recommandé annuellement. Une double dose de vaccin pourrait améliorer la protection antigrippale (34).

Le risque d'infection invasive à pneumocoque est 20 à 40 fois plus élevé chez les patients qui ont eu une greffe de rein comparé à la population générale (35). La vaccination antipneumococcique est recommandée tous les 5 ans après transplantation rénale, avec le vaccin conjugué à 13 valences suivi au moins 2 mois après du vaccin polysaccharidique à 23 valences (36).

La vaccination contre le zona, recommandée en France chez toutes les personnes de 65 à 74 ans, pourrait permettre de diminuer l'incidence de cette complication particulièrement fréquente (36). Ce vaccin vivant atténué est recommandé avant la transplantation, et contre-indiqué au décours.

La vaccination contre l'hépatite B est bien sûr recommandée chez tout patient ayant une maladie rénale chronique. L'immunisation anti-HBs permet aux patients en attente de transplantation rénale de recevoir un greffon dit « dérogatoire VHB », c'est à dire $\mathrm{HBc}+$.

Les principaux vaccins recommandés sont détaillés dans les tableaux 1 et 2.

\section{Particularités des patients infectés par le VIH}

Le risque d'insuffisance rénale terminale est plus élevé chez les patients infectés par le VIH (37). A partir de la fin des années 1990, et l'avènement des trithérapies antirétrovirales, des données encourageantes ont permis de développer la transplantation rénale chez ces patients. Toutes les études concordent sur la bonne survie de ces patients après transplantation rénale, l'absence d'augmentation du risque infectieux, et le bon contrôle virologique du VIH $(38,39)$. En revanche une incidence très élevée de rejet (jusque 30\% à 1 an de greffe), et une moins bonne survie du greffon ont été constatées dans toutes les cohortes des années 2000 (39). Le traitement par inhibiteur de protéase, responsable d'interactions avec les immunosuppresseurs et de sous-dosages en inhibiteurs de la calcineurine, pourrait en être en partie responsable. En effet, chez les patients ne recevant pas d' inhibiteur de protéase, le risque de rejet est diminué, et la survie du greffon améliorée (40). 
Afin d'améliorer l'accès à la greffe des patients infectés par le VIH, des greffons issus de donneurs infectés par le VIH peuvent actuellement, sous certaines conditions, être proposés aux receveurs infectés par le VIH, en Afrique du Sud et aux États-Unis (41). Les résultats sont encourageants.

\section{Conclusion}

Les infections sont donc très fréquentes après transplantation. Le spectre des infections est largement dominé par les pyélonéphrites et les pneumopathies, qui peuvent survenir à n'importe quel moment après la transplantation. La part des infections gastro-intestinales est également majeure, et l'amélioration des techniques diagnostiques devrait permettre une amélioration de leur reconnaissance et de leur prise en charge. Les infections opportunistes, fréquentes, variées, sont une cause importante de morbimortalité.

Les facteurs de risque principaux connus d'infection sont l'âge, le sexe féminin, le diabète, et le rejet. Une meilleure connaissance de ces facteurs de risque, couplée à l'histoire naturelle des infections, devrait permettre de cibler les populations à risque pour optimiser les préventions antiinfectieuses. Des études portant sur les déterminants, le pronostic et l'impact des infections sont encore nécessaires, afin d'améliorer la prise en charge des patients qui ont eu une greffe de rein.

\section{Bibliographie}

1. Alangaden GJ, Thyagarajan R, Gruber SA, Morawski K, Garnick J, El- Amm JM, et al. Infectious complications after kidney transplantation: current epidemiology and associated risk factors. Clin Transplant 2006;20(4):401-9.

2. Fishman JA. Infection in solid-organ transplant recipients. N Engl J Med 2007;357(25):2601-14.

3. San Juan R, Aguado JM, Lumbreras C, Díaz-Pedroche C, López- Medrano F, Lizasoain M, et al. Incidence, clinical characteristics and risk factors of late infection in solid organ transplant recipients: data from the RESITRA study group. Am J Transplant 2007;7(4):964-71.

4. Snyder JJ, Israni AK, Peng Y, Zhang L, Simon TA, Kasiske BL. Rates of first infection following kidney transplant in the United States. Kidney Int 2009;75(3):317-26.

5. Giral M, Pascuariello G, Karam G, Hourmant M, Cantarovich D, Dantal J, et al. Acute graft pyelonephritis and long-term kidney allograft outcome. Kidney Int 2002;61(5):1880-1886.

6. Abbott KC, Swanson SJ, Richter ER, Bohen EM, Agodoa LY, Peters TG, et al. Late urinary tract infection after renal transplantation in the United States. Am J Kidney Dis 2004;44(2):353-62.

7. Pellé G, Vimont S, Levy PP, Hertig A, Ouali N, Chassin C, et al. acute pyelonephritis represents a risk factor impairing long-term kidney graft function. Am J Transplant 2007;7(4):899-907. 
8. Origüen J, López-Medrano F, Fernández-Ruiz M, Polanco N, Gutiérrez E, González E, et al. Should asymptomatic bacteriuria be systematically trated in kideny tranplant recipients ? Result from a randomized controlled trial. Am J Transplant 2016;16(10):2943-2953.

9. Nielsen LH, Jensen-Fangel S, Jespersen B, Østergaard L, Søgaard OS. Risk and prognosis of hospitalization for pneumonia among individuals with and without functioning renal transplants in denmark: a population-based study. Clin Infect Dis 2012;55(5):679-86.

10. Bunnapradist S, Neri L, Wong W, Lentine KL, Burroughs TE, Pinsky BW, et al. Incidence and risk factors for diarrhea following kidney transplantation and association with graft loss and mortality. Am J Kidney Dis 2008;51(3):478-86.

11. Echenique IA, Stosor V, Gallon L, Kaufman D, Qi C, Zembower TR. Prolonged norovirus infection after pancreas transplantation: a case report and review of chronic norovirus. Transpl Infect Dis 2016;18(1):98-104.

12. Roos-Weil D, Ambert-Balay K, Lanternier F, Mamzer-Bruneel M-F, Nochy D, Pothier P, et al. Impact of norovirus/sapovirus-related diarrhea in renal transplant recipients hospitalized for diarrhea. Transplantation 2011;92(1):61-9.

13. Aulagnon F, Scemla A, DeWolf S, Legendre C, Zuber J. Diarrhea after kidney transplantation: a new look at a frequent symptom. Transplantation 2014;98(8):806-16.

14. Marion O, Abravanel F, Lhomme S, Izopet J, Kamar N. Hepatitis E in transplantation. Curr Infect Dis Rep 2016;18(3):8.

15. Manuel O, Kralidis G, Mueller NJ, Hirsch HH, Garzoni C, van Delden C, et al. Impact of antiviral preventive strategies on the incidence and outcomes of cytomegalovirus disease in solid organ transplant recipients: impact of CMV prevention. Am J Transplant 2013;13(9):2402-10.

16. Humar A, Snydman D. Cytomegalovirus in solid organ transplant recipients. Am J Transplant 2009;9:S78-86.

17. Hirsch HH, Knowles W, Dickenmann M, Passweg J, Klimkait T, Mihatsch MJ, et al. Prospective study of polyomavirus type BK replication and nephropathy in renal-transplant recipients. $\mathrm{N}$ Engl J Med 2002; 347:488-496.

18. Hirsch HH, Randhawa P, the AST Infectious Diseases Community of Practice. BK polyomavirus in solid organ transplantation. Am J Transplant 2013;13(s4):179-88. 
19. Schold JD, Rehman S, Kayler LK, Magliocca J, Srinivas TR, Meier-Kriesche H-U. Treatment for BK virus: incidence, risk factors and outcomes for kidney transplant recipients in the United States. Transpl Int 2009;22(6):626-34.

20. Arness T., Pedersen R., Dierkhising R., Kremers W., Patel R. Varicella zoster virus-associated disease in adult kidney transplant recipients: incidence and risk-factor analysis. Transpl Infect Dis 2007;10(4):260-8.

21. Subramanian AK, Morris MI, the AST Infectious Diseases Community of Practice.

Mycobacterium tuberculosis infections in solid organ transplantation: tuberculosis in transplant. Am J Transplant 2013;13(s4):68-76.

22. Torre-Cisneros J, Doblas A, Aguado JM, San Juan R, Blanes M, Montejo M, et al. Tuberculosis after solid-organ transplant: incidence, risk factors, and clinical characteristics in the RESITRA (Spanish Network of Infection in Transplantation) cohort. Clin Infect Dis 2009;48(12):1657-65.

23. Canet E, Dantal J, Blancho G, Hourmant M, Coupel S. Tuberculosis following kidney transplantation: clinical features and outcome. A French multicentre experience in the last 20 years. Nephrol Dial Transplant 2011;26(11):3773-8.

24. Dorman S, Subramanian A, the AST Infectious Diseases Community of Practice. Nontuberculous mycobacteria in solid organ transplant recipients. Am J Transplant 2009;9:S63-9.

25. Pappas PG, Alexander BD, Andes DR, Hadley S, Kauffman CA, Freifeld A, et al. Invasive fungal infections among organ transplant recipients: results of the Transplant-Associated Infection Surveillance Network (TRANSNET). Clin Infect Dis 2010;50(8):1101-1111.

26. Desbois A-C, Poiree S, Snanoudj R, Bougnoux M-E, Sberro-Soussan R, Lanternier F, et al. Prognosis of invasive aspergillosis in kidney transplant recipients: a case-control study. Transplant Direct 2016;2(8):e90.

27. Fishman JA. Prevention of infection due to Pneumocystis carinii. Antimicrob Agents Chemother 1998;42(5):995-1004.

28. Neff RT, Jindal RM, Yoo DY, Hurst FP, Agodoa LY, Abbott KC. Analysis of USRDS: incidence and risk factors for Pneumocystis jiroveci pneumonia. Transplantation 2009;88(1):135.

29. Singh N, Alexander BD, Lortholary O, Dromer F, Gupta KL, John GT, et al. Cryptococcus neoformans in organ transplant recipients: impact of calcineurin-inhibitor agents on mortality. J Infect Dis 2007;195(5):756-64. 
30. Kotton CN, Kumar D, Caliendo AM, Huprikar S, Chou S, Danziger-Isakov L, et al. The Third International Consensus Guidelines on the management of cytomegalovirus in solid organ transplantation. Transplantation 2018;102(6):900-931.

31. Eckerle I, Rosenberger KD, Zwahlen M, Junghanss T. Serologic vaccination response after solid organ transplantation: a systematic review. PLoS One 2013;8(2):e56974.

32. Autran B, Frésard A, Gaudelus J, Hanslik T, Jacquet A, Launay O, et al. Vaccinations des personnes immunodéprimées ou aspléniques. Recommandations. Paris : Haut Conseil de la santé publique ; 2014.

33. Mulley WR, Visvanathan K, Hurt AC, Brown FG, Polkinghorne KR, Mastorakos T, et al. Mycophenolate and lower graft function reduce the seroresponse of kidney transplant recipients to pandemic H1N1 vaccination. Kidney Int 2012;82(2):212-9.

34. Mombelli M, Rettby N, Perreau M, Pascual M, Pantaleo G, Manuel O. Immunogenicity and safety of double versus standard dose of the seasonal influenza vaccine in solid-organ transplant recipients: a randomized controlled trial. Vaccine 2018;36(41):6163-6169.

35. Kumar D, Humar A, Plevneshi A, Green K, Prasad GV, Siegal D, et al. Invasive pneumococcal disease in solid organ transplant recipients--10-year prospective population surveillance. Am J Transplant 2007; 7(5):1209-14.

36. Anon. Calendrier des vaccinations et recommandations vaccinales 2018. Paris : ministère des Solidarités et de la santé ; 2018.

37. Abraham AG, Althoff KN, Jing Y, Estrella MM, Kitahata MM, Wester CW, et al. End-stage renal disease among HIV-infected adults in North America. Clin Infect Dis 2015;60(6):941-9.

38. Stock PG, Barin B, Murphy B, Hanto D, Diego JM, Light J et al. Outcomes of kidney transplantation in HIV-infected recipients. N Engl J Med 2010;363(21):2004-14.

39. Kucirka LM, Durand CM, Bae S, Avery RK, Locke JE, Orandi BJ, McAdams-DeMarco M, et al. Induction immunosuppression and clinical outcomes in kidney transplant recipients infected with human immunodeficiency virus. Am J Transplant 2016;16(8):2368-76.

40. Matignon M, Lelievre JD, Lahiani A, Abbassi K, Desvaux D, Diallo A et al. Low incidence of acute rejection within 6 months of kidney transplantation in HIV-infected recipients treated with raltegravir: the Agence nationale de recherche sur le sida et les hépatites virales (ANRS) 153 TREVE trial. HIV Med 2019; 20(3):202-213. 
41. Muller E, Barday Z, Mendelson M, Kahn D. HIV-positive-to-HIV-positive kidney transplantation-results at 3 to 5 years. N Engl J Med 2015;372(7):613-20. 
Tableau 1. Infections en transplantation rénale : principales vaccinations recommandées avant ou après la transplantation.

\begin{tabular}{|c|c|}
\hline Risque infectieux & Modalités \\
\hline Grippe saisonnière inactivée & Vaccination annuelle \\
\hline Pneumocoque & $\begin{array}{l}\text { Vaccin conjugué à } 13 \text { valences puis vaccin } \\
\text { polysaccharidique à } 23 \text { valences, au moins } 2 \text { mois } \\
\text { après. Tous les } 5 \text { ans }\end{array}$ \\
\hline Diphtérie-tétanos-poliomyélite & Une injection tous les 10 ans \\
\hline Coqueluche acellulaire & Un rappel unique à l'âge adulte \\
\hline Hépatite A & $\begin{array}{l}\text { Deux doses à } 6 \text { mois d'intervalle en cas de : } \\
\text { - co-infection par le virus de l'hépatite B ou C, } \\
\text { patient homosexuel } \\
\text { - voyage en zones d'endémie } \\
\text { - hépatopathie }\end{array}$ \\
\hline Hépatite B & Double dose à $0,1,2,6$ mois \\
\hline Méningocoque $\mathrm{C}$ & Une dose en rattrapage jusqu'à 24 ans \\
\hline $\begin{array}{l}\text { Méningocoque ACYW135 } \\
\text { (conjugué), méningocoque B }\end{array}$ & $\begin{array}{l}\text { Deux doses à } 8 \text { semaines d'intervalle, en cas de : } \\
\text { - asplénie, déficit en complément, traitement par } \\
\text { éculizumab } \\
\text { - pèlerinage à la Mecque, voyage en Afrique en } \\
\text { période épidémique }\end{array}$ \\
\hline $\begin{array}{l}\text { Haemophilus influenzae b } \\
\text { (conjugué) }\end{array}$ & Une dose unique en cas d'asplénie \\
\hline Papilloma virus humain (HPV) & Filles et garçons de 9 à 19 ans, trois doses \\
\hline
\end{tabular}


Hommes ayant des relations avec des hommes :

jusqu'à l'âge de 26 ans 
Tableau 2. Infections en transplantation rénale : principales vaccinations (vaccins vivants atténués) recommandées uniquement avant la transplantation, contre-indiquées au décours.

\begin{tabular}{|l|l|}
\hline Risque infectieux & Modalités \\
\hline Rougeole & $\begin{array}{l}\text { Deux doses en tout pour les personnes nées après 1980 ou } \\
\text { séronégatives }\end{array}$ \\
\hline Varicelle & $\begin{array}{l}\text { Deux doses espacées de } 4 \text { à } 8 \text { semaines chez les personnes } \\
\text { séronégatives }\end{array}$ \\
\hline Zona & Une dose chez les personnes âgées de 65 à 74 ans \\
\hline Fièvre jaune & Une injection en cas de sérologie négative et de voyage en \\
& zones d'endémie prévu \\
\hline
\end{tabular}

